\title{
Transition Stages between Order and Disorder in Condensed Phases
}

$\mathrm{O}^{\mathrm{N}}$ NE necessary stage in the learning of physical chemistry is that in which the crystalline state is represented as one of perfect order-one molecule to each lattice point, only disturbed by the thermal motion, a quivering of the structure as a whole - and the liquid and gaseous states as entirely disorderly, the equation of van der Waals being used to demonstrate that a disorderly assembly of molecules can undergo a two-phase condensation. This was the stage which the education of physical chemists as a whole had reached a few years ago; but now the gradual accumulation of new knowledge about the detailed molecular structure of condensed matter is forcing us to learn lessons of which such pioneers as Boltzmann and Mie were in principle aware by the turn of the century. They had perceived already, in particular from specific heats and densities, that molecules in a liquid must undergo very much the same motions as in the solid state. Now we are learning that there may be a great deal of order in the liquid and a great deal of disorder in the crystalline state, and it is even by no means so obvious as once it seemed that liquid and crystalline solid must form two sharply distinct phases.

This is the state of knowledge which makes most opportune the German Bunsen Society's second discussion meeting, which was organized by Prof. C. Wagner at Darmstadt on October 28-29. The main purpose which this meeting served was to bring together very fully and systematically all the methods by which the molecular structure of condensed matter can be investigated, and the results, in respect of departure from the crude classical picture, which have been obtained.

Any of the following sorts of disorder may occur in the crystal: (1) unlike atoms, especially if not too unlike, can exchange places; (2) lattice spaces may be unoccupied; (3) molecules (atoms, ions) may be present at wrong sites (interlattice spaces); (4) molecules or ionic groups may be wrongly oriented, or may rotate.

(1) is a common occurrence in alloys. In such cases, for example, $\beta$-brass, there is at low temperatures a 'superlattice' of orderly alternation of copper and zinc atoms, which becomes increasingly disordered on heating and finally vanishes in a transition of second order to a random distribution, while the ground-structure, disregarding the differences between copper and zinc atoms, remains substantially unaltered. Borelius brought together a great deal of experimental data on these place change transitions in alloys, which have aroused revived interest since Bragg and Williams offered an explanation of them. The basic principle of this explanation is that a small amount of disorder weakens the potential maintaining the structure, so that each further step towards increasing disorder becomes easier and easier, a fact which reveals itself in a continuously accelerating rate of change with rising temperature, and a high and rising specific heat, which drops abruptly when the disorder is completed. This is known as a co-operative change.
A number of very sensitive optical, electrical, and photo-electric methods of investigating small amounts of disorder in ionic crystals have been developed by Pohl and his collaborators; one of these, the measurement of electrolytic conductivity in the solid, has been recently applied by Wagner to give quantitative determinations of the disorder present, following a theoretical attack on the problem in conjunction with Schottky. This is particularly sensitive to (2): a vacant ionic space behaves as a mobile ion of the opposite charge. To obtain additional information he purposely produces vacancies by adding impurity of another ionic type, for example, $\mathrm{CdCl}_{2}$ to $\mathrm{AgCl}$. Vacant spaces may be formed spontaneously in something like vapour concentration, ions of both signs being missing; in greater concentration as the accompaniment of (3), that is, when some ions are displaced in the crystal ; in almost any concentration when impurities of another ionic type are added, or when ions of variable valency are present; or finally in stoichiometric amount when the numbers of lattice points for cations and anions is not in the same ratio as the numbers of ions. Usually the large anions form a substantially perfect simple lattice while the cations form a more disordered one in the interstices. For example, in silver bromide the bromine ions form a cubical close-packed lattice. At room tem. perature, all but about one in a thousand of the silver ions occupy a similar lattice in the octahedral interstices of this one, but by $400^{\circ} \mathrm{C}$. (the melting point is $416^{\circ} \mathrm{C}$.) no less than 16 per cent of them are displaced into the smaller tetrahedral interstices. $\alpha$-AgI provides a more extreme case: in this the anions form a looser, body centred cubic structure, and the silver ions are distributed practically at random over forty-two possible sites in each unit cell-in fact they may well be regarded as forming a positively charged liquid permeating the anionic crystal, and they give rise to a very large electrolytic conductivity, which is practically unaffected when the crystal melts. Such gross disorder as the latter is of course accessible to $\mathrm{X}$-ray investigation, and this method has produced a multiplicity of examples, much more complex than these simple ones, and of a variety which would soon have become overwhelming without the newly devised systematic classification used by Laves in reporting on this field.

Following these two accounts of experimental results, their theoretical background in statistical mechanics was painted in by Schottky, with his usual meticulous attention to fundamental detail. But even so there remain many difficulties to be disposed of in this complex field of theory. Schottky and Laves suggested that a complete description can be given in terms of the extent of occupation of a number of interpenetrating partial lattices; but Debye in discussion indicated that it is also necessary to consider how the occupation of any particular point in one partial lattice influences the probability of occupation of neighbouring points in that and others. This is of course implicit in the theory of co-operative changes, which Schottky presented in Kirkwood's approximation. 
After these sorts of disorder characteristic of crystals composed of monatomic elementary particles we come to another, (4) above, which only enters where independent covalently bound groups of atoms are present : here we find a characteristic group of phenomena which may be confidently attributed to rotation or not fully determinate orientation of these groups within the crystal. Where polar molecules are present, we find large dielectric constants. Pauling predicted this for $\mathrm{HCl}$ in 1930, and its confirmation enforced the acceptance of a hypothesis which had been received very sceptically when first proposed by Simon to account for specific heat anomalies; but it was certainly a shock to all preconceived notions when Yager and Morgan found that the dielectric constant of molten camphor does not alter when it freezes, only dropping to a low value in a transition $120^{\circ}$ below the melting point, in spite of the size and rigidity of the molecule. In many cases we find specific heats considerably exceeding the values of similar monatomic substances (for example, $\mathrm{NH}_{4} \mathrm{Cl}$ compared with $\mathrm{NaCl}$ ). The onset of 'rotation' as temperature rises is commonly marked by a range over which the specific heat rises to very large values to produce a $\lambda$-point transition -this is the characteristic sign of a co-operative effect : it means that the rotation of a few molecules reduces the potential hindering the rotation of others, and the theory of it is essentially the same as that of the co-operative place-change transitions in alloys.

'The word 'rotation', incidentally, should be interpreted rather cautiously, for in many respects the same properties result if each molecule has a choice between two or more possible orjentations. The actual extent to which the rotation is hindered must vary very widely from case to case. For polar molecules it can be actually determined when dielectric dispersion or absorption is observed, and may be declared to be low when it is not. Rough estimates can also be made from the specific heat curves, if the necessary data to reduce these to constant volume are available. Usually they are not, and to meet this Eucken made bold use of empirical rules in preparing what was in the outcome a very instructive review of the changes in specific heat which accompany 'rotational' transitions. It may be presumed that the empirical rules are least reliable precisely in the interesting regions where there are large co-operative structural contributions to the specific heat, but this does not weaken the conclusion that in many cases something like free rotation is attained. The X-ray method is unable to give much information for the smaller molecules with only one heavy atom, but reveals rotation of paraffin chains, as Pauling pointed out in his first paper on this subject, and has recently been applied by Hassel to cyclo-hexanol and its derivatives. It will be interesting to see the result of an X-ray study of camphor.

The discussion of disorder arising in the crystal was completed by Gerlach's report on the ferromagnetic Curie point, the prototype of co-operative second order transformations, and a brief account sent by Scherrer of the behaviour of Rochelle salt, its dielectric analogue.

The rest of the discussion was devoted to the complementary case of the order which is present in substances which are not crystals, that is, in liquids, crystalline liquids, glasses and rubber. Debye was chiefly concerned to explain all the known lines of attack by which we can discover the extent of the quasi-crystalline structure present in liquids. The specific heat at constant volume of monatomic liquids indicates that the atoms possess considerable potential energy, not very different from that in the solid. The fine structure of the Rayleigh line in scattered light reveals the same. Light is only scattered from irregularities of density in the body having the same scale as its wave-length. Hence the molecular structure has no influence, but the scattering irregularities are those produced by the thermal motion. In a crystal it is known that the close coupling of the motions of neighbouring molecules causes these motions to take the form of trains of sound waves, and the observed scattered light is reflected from these wave-trains according to the Bragg reflection law. A doublet structure results as a Doppler effect from wave-trains going in opposite directions. It was at one time supposed that the liquid gave a triplet structure owing to its intermediary state between gas and crystal, but it appears that this is only a secondary result of the large difference between $C_{p}$ and $C_{v}$ in most liquids : in water at $4^{\circ}$ Ramm found only a doublet. There is, however, an appreciable broadening of the component lines, which as Zernike remarked, indicates that the wave-trains are shorter in the liquid, showing higher damping.

The damping of supersonic waves in liquids can be directly measured, and is found to be much larger than can be explained by the viscosity and thermal conduction. It can be explained if part of the com. pressibility of the liquid involves molecular rearrangement, having a relaxation time of the order of magnitude $10^{-10}$ sec. This method has not been properly exploited yet. The newest and best method, of measurement again uses the diffraction of light, in this case from artificially produced wave-trains.

To find the structure on a molecular scale we must use radiations of much shorter wave-length. The blurred rings in the $\mathrm{X}$-ray diffraction pattern of liquids discovered by Debye and Scherrer and given their full interpretation by Prins and Zernike tell us the average value of the product $\delta_{1} \delta_{2}$ for any desired value of $r$, where $\delta_{1}$ and $\delta_{2}$ are the deviations from the average electron density at any two points a distance $r$ apart from one another. Though this does not suffice to give us a complete unambiguous picture of the structure, it tells us a great deal about it, and allows us to reject false proposals. We find that the arrangement of atoms in liquid mercury is more nearly crystalline than random, rather like a bag of marbles, and that long-shaped molecules favour parallel positions, like a bag of nails. Along any line drawn in imagination through the substance we shall find a regular fluctuation in density, with much the average amplitude and period in the liquid as in the crystal : the great difference is that this periodic fluctuation is coherent over great distances in the crystal, but only over short distances in the liquid.

When dipole molecules are present, we can again use the dielectric properties to gain information about the freedom of orientation of molecules : the dielectric constant of a dipole liquid is often much less than it would be if the molecules exerted no influence on each other's orientation. Although the Debye theory of hindering potential is doubtless not general enough, and must give place to some such description as Onsager's, it directed attention to this source of information and provided the first quantitative treatment, sufficient to rule out any suggestion 
that the molecules in a liquid rotate freely, unhindered by their neighbours. The structure is quasicrystalline with regard to orientation as well as to translation.

In the concluding reports Kast dealt with anisotropic liquids, Jenckel with glasses and Wittstadt with rubber. The transformation of such a liquid as $p$-azoxyanisole to the nematic state, at which we know from the optical properties that some 30 per cent of the rod-shaped molecules suddenly become roughly parallel to one another over fairly wide regions while the $\mathrm{X}$-ray scattering diagram scarcely alters and the fluidity actually increases, is probably one of the simplest of all transitions of a quasicrystalline to a crystalline state. Kast's presentation of the facts was very favourable to Frank's theoretical description of the change. Jenckel concerned himself mainly with the transformation point of glasses. This is the temperature at which the viscosity becomes so high (about $10^{13}$ ) that molecular rearrangement no longer takes place in times compatible with human patience. Below this temperature the coefficient of expansion and the specific heat are much smaller. It is chiefly interesting in the present connexion for showing that molecular rearrangement in the liquid contributes largely to these properties above that temperature. Rubber provides a superb example of the way in which the various lines of attack described above, thermal, Röntgenographic, dielectric and statistical mechanical, have been applied to the elucidation of a peculiarly complex case. The presence together of Kuhn, Meyer and Thiessen ensured a lively discussion.

Prof. Wagner and the Bunsen-Gesellschaft are to be congratulated on the success of this, the second Diskussionstagung which it has organized after the Faraday Society model, circulating all main contributions in full beforehand.

F. C. Frank,

\section{Science and the Army}

$\mathrm{A}^{\mathrm{T}}$ T the Science Museum, South Kensington, there has been arranged a special War Office Exhibition which, for a period of three months, gives visitors an opportunity of acquainting themselves with the numerous points at which science comes into contact with the work of the British Army. It will probably surprise many to learn that, at the several training centres for boys who enter at fourteen years of age, technical instruction, both practical and theoretical, is given which compares favourably with that obtained by the average apprentice in civil life. At the Military College of Seience, Woolwich, this reaches its highest development in the training for the grade of artificer, Royal Artillery, the course for which extends over five years and produces men of high technical skill. Mechanization accounts for a great increase in the engineering requirements of Army services, and the extent of this will be realized from the exhibits showing the preparations for the driving and maintenance of transport vehicles and tanks and for the repairs carried out in the heavy workshops operated by the Royal Engineers and Royal Army Service Corps. The high stage of development reached in the signals and wireless services is demonstrated and here, to a large extent, the apparatus has to be specially designed for the conditions of use in the field, though, so far as possible, commercial forms are adopted.

In certain branches, it will be seen that the Army has been leading the way, and this is notably the case in the making of maps. The exhibit shows that the War Office, breaking away from the Continental practice, has developed a comparatively simple method of aerial surveying, and the various stages of making the types of maps which can be produced in the field are illustrated. The manifold services of the Royal Engineers can be seen in exhibits of different kinds of bridges made and used in war, and in examples of demolitions, water supply arrangements with well-sinking and distribution, and such work as the drainage schemes carried out in conjunction with Royal Army Medical Corps anti- malarial measures at places like Singapore. Medical science is represented by models of field disinfectors, water purifiers and the latest type of small cooker for use in tanks.

In the Air Defence Section some highly scientific exhibits are to be seen. The sound locator is based on the principle of human hearing whereby, when waves of sound reach the ear, the head is turned until they come equally to each ear. So, the locator is turned until its trumpets, one pair for bearing and one pair for elevation, give the position of the source of the sound. The Vickers predictor is, in a like sense, a pair of mechanical eyes. In addition to ranging its target, it gives the bearing, the elevation, and the right fuse-setting, making allowance for trajectory curve, wind, and even for variation in barometric pressure in the upper air. Its information is transmitted electrically to dials on the guns, and all that the men on the gun have to do is to keep mechanical pointers in line with the electrical pointers.

Research in Army matters is much more coordinated than it is in civil affairs. Development in one direction has immediate repercussions in others -if, for example, a gun of higher rate of fire is visualized, questions as to ammunition, supplies, and possibly man-power will have to be dealt withand consequently the research organization is very broadly based as the exhibition will show. A most interesting exhibit is the R. D. Camera which films shells as they are actually fired. The Ballistic Section also shows the Brown-Hall photographic lorry which enables this filming to be done in the field and the cascade thyratron which measures bullet velocities when 'bursts' are fired. The Chemical Research Branch at Porton and the Experimental Establishment at Biggin Hill are also represented, and this section of the Exhibition conveys an impression of the unceasing scientific inquiry that is conducted by the War Office to ensure that the highest efficiency of man and material may be attained in the requirements of the Service. 